\title{
CUIDADOS DE ENFERMAGEM NAS COMPLICAÇÕES NO PÓS-OPERATÓRIO DE CIRURGIA DE REVASCULARIZAÇÃO DO MIOCÁRDIO
}

\section{NURSING CARE IN POSTOPERATIVE COMPLICATIONS OF MYOCARDIAL REVASCULARIZATION SURGERY}

\section{CUIDADOS DE ENFERMERÍA EN LAS COMPLICACIONES EN EL POST-OPERATORIO DE CIRUGÍA DE REVASCULARIZACIÓN DEL MIOCARDIO}

\author{
Liliane de Lourdes Teixeira Silva ${ }^{1}$ \\ Luciana Regina Ferreira da Mata $^{2}$ \\ Adriana Ferreira Silva ${ }^{3}$ \\ Jessica Campos Daniel ${ }^{3}$ \\ Ana Flávia Leite Andrade \\ Emylle Thayssa Mendonça Santos ${ }^{3}$
}

\begin{abstract}
Como citar este artigo: Silva LLT, Mata LRF, Silva AF, Daniel JC, Andrade AFL, Santos ETM. Cuidados de enfermagem nas complicações no pós-operatório de cirurgia de revascularização do miocárdio. Rev baiana enferm. 2017;31(3):e20181.

Objetivo: descrever as complicações e os cuidados de enfermagem ofertados aos pacientes no pós-operatório da cirurgia de revascularização do miocárdio. Método: estudo descritivo retrospectivo, com abordagem quantitativa, realizado mediante análise de 80 prontuários de indivíduos submetidos à cirurgia de revascularização do miocárdio entre janeiro e dezembro de 2014. Resultados: dentre as complicações encontradas no pós-operatório, destacaram-se as complicações pulmonares $(82,5 \%)$ e as cardíacas (30\%). Notou-se que a monitoração do equilíbrio de líquido e dos sinais vitais, seguido pelo reconhecimento na alteração da pressão sanguínea foram os cuidados de enfermagem mais ofertados. Conclusões: a monitoração dos sinais vitais, o reconhecimento da alteração da pressão sanguínea, a monitoração do equilíbrio de líquidos e dos padrões respiratórios para bradipneia, taquipneia e hiperventilação foram os cuidados de enfermagem mais ofertados aos pacientes no período pós-operatório da cirurgia de revascularização do miocárdio.
\end{abstract}

Descritores: Cirurgia torácica. Complicações pós-operatórias. Cuidados de enfermagem. Enfermagem.

Objective: to describe the complications and the nursing care offered to patients in the postoperative period of myocardial revascularization surgery. Method: a retrospective descriptive study with quantitative approach was carried out through the analysis of 80 medical charts of individuals submitted to myocardial revascularization surgery between January and December 2014. Results: pulmonary (82.5\%) and cardiac (30\%) were the most frequent complications found in the postoperative period. It was observed that monitoring fluid balance and vital

\footnotetext{
Doutora em Ciências da Saúde. Professora adjunto I do curso de enfermagem da Universidade Federal de São João Del Rei. São João Del Rei, Minas Gerais, Brasil. lilanets@ufsj.edu.br

Doutora. Professora Adjunto II da Universidade Federal de São João Del Rei. Editora Chefe da Revista de Enfermagem do Centro-Oeste Mineiro. Docente do Programa de Pós-graduação em Enfermagem da Universidade Federal de São João Del Rei - nível mestrado acadêmico. Docente do Programa de residência em Enfermagem na Atenção Básica/Saúde da Família, Universidade Federal de São João Del Rei/Campus Centro-Oeste Dona Lindu. Membro da Câmara Técnica de Sistematização da Assistência de Enfermagem do Conselho Regional de Enfermagem de Minas Gerais. Líder do Núcleo de Pesquisas e Estudos em Cuidar em Enfermagem e em Saúde, Universidade Federal de São João Del Rei. Membro do grupo de pesquisa Enfermagem e Comunicação, Escola de Enfermagem de Ribeirão Preto, Universidade de São Paulo e do grupo Family Health and Illness da Universidade do Minho, Portugal.

Enfermeiras.
} 
signs, followed by detection of altered blood pressure were the most frequently provided nursing care measures. Conclusions: monitoring of vital signs, recognition of changes in blood pressure, monitoring of fluid balance and of respiratory patterns for bradypnea, tachypnea, and byperventilation were the most frequently offered nursing care measures to patients in the postoperative period of myocardial revascularization surgery.

Descriptors: Thoracic surgery. Postoperative complications. Nursing care. Nursing.

Objetivo: describir las complicaciones y los cuidados de enfermería ofertados a los pacientes en el post-operatorio de la cirugía de revascularización del miocardio. Método: estudio descriptivo retrospectivo, con enfoque cuantitativo, realizado mediante análisis de 80 prontuarios de individuos sometidos a la cirurgia de revascularización del miocardio entre enero y diciembre de 2014. Resultados: dentro de las complicaciones encontradas en el postoperatorio, se destacaron las complicaciones pulmonares (82,5\%) y las cardiacas (30\%). Se notó que el monitoreo del equilibrio de líquido y de los signos vitales, seguido por el reconocimiento en la alteración de la presión sanguinea fueron los cuidados de enfermería más ofertados. Conclusiones: el monitoreo de los signos vitales, el reconocimiento de la alteración de la presión sanguinea, el monitoreo del equilibrio de líquidos y de los padrones respiratorios para bradipnea, taquipnea y hiperventilación fueron los cuidados de enfermería más ofertados a los pacientes en el periodo post-operatorio de la cirugía de revascularización del miocardio.

Descriptores: Cirugía torácica. Complicaciones post-operatorias. Cuidados de enfermería. Enfermería.

\section{Introdução}

As doenças crônicas não transmissíveis englobam um conjunto de patologias, como as doenças cardiovasculares (DCV), neoplasias, doenças respiratórias crônicas e diabetes mellitus, as quais destacam-se pelo elevado padrão de morbidade e mortalidade. Além disso, possuem etiologia múltipla, associada a deficiências e incapacidades funcionais, que são potencializadas por fatores socioeconômicos, culturais e ambientais ${ }^{(1)}$. As DCV têm o maior impacto epidemiológico e são responsáveis por cerca de 30\% de todas as mortes no mundo ${ }^{(2)}$. Diante disso, as cardiopatias representam um grande problema de saúde pública, sendo imprescindível o uso de métodos diagnósticos, terapêuticos e preventivos capazes de interromper sua progressão e evitar seu surgimento ${ }^{(3)}$.

A cirurgia cardíaca é uma alternativa para prolongar a vida desses pacientes e reduzir a morbimortalidade por doenças circulatórias. É realizada somente quando o tratamento clínico não é capaz de proporcionar a cura e/ou melhoria da qualidade de vida do paciente ${ }^{(3)}$. As cirurgias cardíacas são classificadas em corretoras, reconstrutoras e substitutivas. Dentre elas, as mais comuns são as reconstrutoras, especialmente a revascularização do miocárdio (3). A cirurgia de revascularização do miocárdio (CRVM) possui como objetivo a correção da isquemia miocárdica em consequência da obstrução das artérias coronárias, buscando alívio dos sintomas e a melhora da qualidade de vida, assim como o retorno mais precoce do paciente às atividades laborais e o aumento da expectativa de vida $^{(4)}$. Contudo, há possibilidade de o paciente desenvolver complicações no pós-operatório de CRVM, como alterações cardíacas, pulmonares, cerebrovasculares, infecciosas e renais ${ }^{(5)}$.

As complicações pós-operatórias da CRVM são amplamente discutidas na literatura e sua incidência varia de acordo com fatores individuais, como idade, hábitos de vida, comorbidades associadas e situação clínica no momento da cirurgia, além de questões relacionadas ao processo cirúrgico, como tempo de duração da cirurgia, uso de Circulação extracorpórea, agentes anestésicos utilizados e complicações no período transoperatório.

A identificação precoce das principais complicações no pós-operatório de CRVM permite ao enfermeiro atuar de forma segura e humanizada e assim traçar uma estratégia de cuidado que previna danos, melhore o prognóstico do paciente e reduza o tempo de permanência no ambiente hospitalar ${ }^{(6)}$. Conhecer os cuidados prestados aos pacientes pode indicar qual o 
caminho seguido pela equipe de enfermagem na assistência ao usuário e se há necessidade de realizar ajustes na assistência ofertada.

Desta maneira, este estudo pretende descrever as complicações do pós-operatório de CRVM em pacientes internados em um hospital de ensino do interior de MG e identificar quais os principais cuidados realizados para resolução dessas. Reconhecer o padrão de complicações e cuidados poderá auxiliar a equipe de enfermagem na construção de um instrumento de trabalho visando intervenções precoces e efetivas para essa população.

O objetivo deste estudo é descrever as complicações e os cuidados de enfermagem ofertados aos pacientes no pós-operatório da cirurgia de revascularização do miocárdio.

\section{Método}

Trata-se de um estudo descritivo retrospectivo, realizado mediante análise de prontuários. A pesquisa foi realizada em um hospital de grande porte do interior de Minas Gerais. A Instituição de Saúde pesquisada é referência em atendimento para urgência, emergência e procedimentos eletivos, atendendo os pacientes do Sistema Único de Saúde (SUS), convênios ou particular.

Foram consultados os prontuários de todos os pacientes submetidos à CRVM entre o período de janeiro e dezembro de 2014, o que totalizou 80 prontuários. Os dados foram coletados no período de junho e julho de 2015. O critério de inclusão para o estudo consistiu em todos os pacientes que foram submetidos à cirurgia de revascularização do miocárdio. Já o critério de exclusão foi a idade do paciente inferior a 18 anos.

Para o levantamento dos dados foi utilizado formulário elaborado pelas pesquisadoras, que contém as variáveis sociodemográficas - sexo, idade, raça, procedência e estado civil - e variáveis clínicas - que identificam se o paciente possui história de hipertensão arterial sistêmica, diabetes mellitus, dislipidemia, doença pulmonar obstrutiva crônica, doença renal, infarto agudo do miocárdio prévio, doença cerebrovascular, tabagismo e o índice de massa corporal. O formulário também apresenta uma lista de complicações e possíveis cuidados de enfermagem relacionados, que foram identificados na literatura nacional ${ }^{(3,5,7-11)}$.

A análise dos dados foi realizada por meio da análise descritiva, com o apoio do programa SPSS 17.0. Os resultados foram apresentados em tabelas de distribuição de frequência. $O$ projeto foi submetido ao Comitê de Ética em Pesquisa da Universidade Federal de São João Del Rei e da Instituição coparticipante, sendo aprovado sob Parecer n. 1.011.644, de 4/3/2015. Foram seguidos os princípios de privacidade e confidencialidade, conforme previsto na Resolução n. 466/2012, que regulamenta a pesquisa em seres humanos.

\section{Resultados}

Dos 80 participantes, $81,2 \%$ eram do sexo masculino, 35,3\% apresentaram idade entre 51 a 59 anos, $41,5 \%$ encontravam-se entre a faixa etária de 60 a 69 anos de idade e 5\% tinham idade entre 81 e 84 anos. Quanto à cor, 25,0\% dos pacientes declararam-se brancos e 2,5\% negros. A maioria dos pacientes residia na zona urbana. Em relação à situação conjugal, a maioria era casado (61,3\%), seguido dos solteiros $(25,0 \%)$, $6,2 \%$ eram divorciados e $5,0 \%$ viúvos.

Em relação à história clínica anterior à cirurgia, 77,5\% possuíam histórico de hipertensão arterial sistêmica, 38,8\% eram portadores de diabetes mellitus, 38,8\% apresentavam dislipidemia, $10,0 \%$ dos pacientes manifestaram doença pulmonar obstrutiva crônica, 3,8\% dos pacientes tinham doença renal, sendo 1,3\% insuficiência renal crônica e 2,5\% insuficiência renal aguda. $\mathrm{O}$ infarto agudo do miocárdio esteve previamente presente em $46,3 \%$ dos pacientes.

Quanto aos hábitos de vida, 65\% dos pacientes eram tabagistas e 18,8\% apresentavam histórico de etilismo quando foram submetidos à internação. No tocante ao índice de massa corpórea, 1,3\% dos pacientes eram de baixo peso, $21,3 \%$ apresentavam peso normal, $12,5 \%$ encaixavam-se no grupo sobrepeso e $2,5 \%$ demonstraram ter obesidade grau I.

Do total de cirurgias realizadas, em $96,3 \%$ foi utilizada a circulação extracorpórea (CEC). Em 
63,8\% o tempo de CEC esteve entre 40 e 100 minutos; em 26,7\%, entre 101 e 160 minutos; e apenas $1,3 \%$ utilizaram a CEC por mais de 161 minutos.

Dentre as complicações do aparelho circulatório encontradas no pós-operatório destacaram-se as arritmias, ocorridas em 30\% dos pacientes. Dentre estas, 45\% apresentavam taquicardia sinusal, 29,1\% bradicardia, 16,6\% fibrilação atrial, 4,16\% flutter atrial.

Tabela 1 - Principais complicações no pós-operatório da CRVM. Divinópolis, Minas Gerais, Brasil $-2015$

\begin{tabular}{|c|c|c|}
\hline Variáveis & $\mathbf{n}$ & $\%$ \\
\hline \multicolumn{3}{|l|}{ Complicações Cardíacas } \\
\hline Arritmias & 24 & 30,0 \\
\hline Dor torácica & 19 & 23,8 \\
\hline Instabilidade hemodinâmica & 10 & 12,5 \\
\hline Infarto Agudo do Miocárdio & 5 & 6,3 \\
\hline \multicolumn{3}{|l|}{ Complicações Cerebrovasculares } \\
\hline Alteração do comportamento & 18 & 22,5 \\
\hline Acidente Vascular Cerebral & 1 & 1,3 \\
\hline \multicolumn{3}{|l|}{ Complicações Pulmonares } \\
\hline Ventilação mecânica superior a 24 horas & 17 & 21,3 \\
\hline Insuficiência Respiratória & 12 & 15,0 \\
\hline Broncoespasmo & 4 & 5,0 \\
\hline Pneumotórax & 3 & 3,8 \\
\hline Hipóxia & 1 & 1,3 \\
\hline \multicolumn{3}{|l|}{ Complicações Renais } \\
\hline Diminuição débito urinário & 14 & 17,5 \\
\hline Necessidade de diálise & 3 & 3,8 \\
\hline \multicolumn{3}{|l|}{ Complicações Infecciosas } \\
\hline Pneumonia & 11 & 14,2 \\
\hline Urinária & 1 & 1,3 \\
\hline Mediastinite & 1 & 1,3 \\
\hline
\end{tabular}

Fonte: Elaboração própria.

Outras complicações cardíacas surgiram no pós-operatório e foram descritas como atrito pericárdico (16,2\%), hipotermia, hipertermia, insuficiência cardíaca e hipotensão (2,5\%) cada uma, além de inversão da onda $\mathrm{T}$, sugestiva de isquemia miocárdica, encontrada em 4,16\% dos pacientes. Dentre as demais complicações pulmonares, 14 participantes apresentaram alterações pulmonares que não estavam descritas no formulário, quais sejam: taquipneia $(28,5 \%)$, derrame pleural $(28,5 \%)$, atelectasia (14,2\%), hipercapnia $(7,14 \%)$ e edema agudo de pulmão $(7,14 \%)$.

Tabela 2 - Principais intervenções de enfermagem ofertadas aos pacientes submetidos à CRVM. Divinópolis, Minas Gerais, Brasil - 2015

\begin{tabular}{|c|c|c|}
\hline Variáveis & $\mathbf{n}$ & $\%$ \\
\hline \multicolumn{3}{|l|}{ Complicações Cardíacas } \\
\hline Monitoração dos sinais vitais & 51 & 63,7 \\
\hline Reconhecimento na alteração da pressão sanguínea & 48 & 60,0 \\
\hline Monitoração do equilíbrio de líquidos & 40 & 50,0 \\
\hline
\end{tabular}


Tabela 2 - Principais intervenções de enfermagem ofertadas aos pacientes submetidos à CRVM. Divinópolis, Minas Gerais, Brasil - 2015

\begin{tabular}{l|c|c}
\hline Variáveis & n & \% \\
\hline Complicações Cerebrovasculares & & \\
Monitoração dos sinais vitais & 17 & 21,2 \\
Reconhecimento na alteração da pressão sanguínea & 16 & 20,0 \\
Medicação conforme o necessário & 15 & 18,7 \\
Complicações Pulmonares & & \\
Monitoração dos padrões respiratórios, para bradipneia, taquipneia e hiperventilação & 29 & 36,2 \\
Monitoração da frequência, ritmo, profundidade e do esforço das respirações & 24 & 30,0 \\
Monitoração do aumento de agitação, ansiedade e dispneia & 17 & 21,2 \\
Complicações Renais & & \\
Manutenção de um registro minucioso da ingestão e da eliminação & 10 & 12,5 \\
Monitoração dos sinais vitais & 10 & 12,5 \\
Oferecimento de líquidos quando necessário & 6 & 7,5 \\
Complicações Infecciosas & \multicolumn{2}{c}{} \\
Uso de luvas conforme exigência das precauções padronizadas & 2 & 2,5 \\
Manutenção do ambiente asséptico na troca de equipos e frascos & 1 & 1,2 \\
Exame da condição de qualquer incisão cirúrgica/ferida & 1 & 1,2 \\
\hline
\end{tabular}

Fonte: Elaboração própria.

Os cuidados que menos se destacaram para complicações cardíacas durante a internação foram a observação de sinais e sintomas de débito cardíaco diminuído (12,5\%), orientação sobre a importância de relatar imediatamente qualquer desconforto no peito $(6,3 \%)$ e observação do abdome em busca de indicadores de perfusão reduzida $(1,3 \%)$.

Para as complicações cerebrovasculares, os cuidados prestados com menor frequência aos pacientes foram limitação do número de cuidadores $(1,3 \%)$, monitoração do tempo da protrombina e o tempo parcial da tromboplastina $(1,3 \%)$, além da monitoração da pressão intracraniana do paciente e a resposta neurológica às atividades de cuidado (1,3\%).

Em relação aos cuidados para as complicações pulmonares, os menos ofertados foram monitoração da capacidade do paciente de tossir de forma efetiva $(8,8 \%)$, observação de mudanças na $\mathrm{SaO}_{2}$ e $\mathrm{SvO}_{2}$, no $\mathrm{CO}_{2}$ expirado e nos valores da gasometria arterial $(8,8 \%)$ e monitoração dos valores das provas de função pulmonar, particularmente capacidade vital, força inspiratória máxima, volume expiratório forçado/capacidade vital forçada $(1,3 \%)$.
Alterações no aparelho urinário estiveram presentes em 21,3\% dos pacientes, sendo que os cuidados menos frequentes a esses pacientes foram avaliação diária do peso do paciente (2,5\%), monitoração da perda de líquidos (1,3\%) e instrução de restrição hídrica, quando adequado (1,3\%). Quanto às complicações infecciosas, destacou-se a presença de um quadro de mediastinite registrado. Dentre os cuidados aos pacientes com complicações infecciosas que menos se destacaram, observou-se o uso de luvas estéreis de forma adequada $(1,2 \%)$, limpeza da pele do paciente com agente antibacteriano $(1,2 \%)$ e monitoração de sinais e sintomas sistêmicos e locais de infecção (1,2\%).

\section{Discussão}

A literatura aponta maior prevalência do sexo masculino, tabagistas e de idosos nas CRVM $^{(4,10)}$. Destaca também a relação entre a presença de comorbidades, como Hipertensão Arterial, Diabetes Mellitus, Dislipidemia e a necessidade de cirurgia. Os dados encontrados nessas pesquisas corroboram os achados deste estudo, tendo em vista que grande parte dos pacientes submetidos à CRVM 
eram homens em faixa etária superior a sessenta anos, tabagistas e que possuíam diagnóstico prévio das comorbidades listadas acima. Ressalta-se que a presença de tais características individuais e clínicas atuam como fator de risco para o surgimento de complicações no período pós-operatório ${ }^{(4-5,12-14)}$.

Investigações identificaram que a maior parte dos pacientes que necessitaram ser submetidos à CRVM encontravam-se com IMC acima de $25 \mathrm{~kg} / \mathrm{m}^{2}$, isto é, em sobrepeso ${ }^{(12,15)}$. Nos dados levantados no presente estudo, esta associação não pôde ser realizada, uma vez que não houve registro do IMC em todos os prontuários avaliados.

Um dos fatores que podem aumentar a morbimortalidade e ocasionar complicações no pós-operatório, principalmente em idosos, é o tempo de circulação extracorpórea (CEC). A CEC prolongada, além de causar a Síndrome da Resposta Inflamatória Sistêmica (SIRS), predispõe a complicações pós-operatórias, principalmente respiratórias $^{(13-14)}$. $\mathrm{Na}$ maioria dos pacientes pesquisados foi realizada a CRVM com uso da CEC, mas o atual estudo não teve como objetivo realizar a associação entre CEC e complicações pós-operatórias. Contudo, outra investigação demonstra associação de CEC e complicações, como a presença de dor, oligúria, hiperglicemia, hipotensão, arritmias, náuseas e febre ${ }^{(16)}$. Destaca-se a necessidade de o enfermeiro reconhecer previamente essas possíveis complicações relacionadas ao uso da CEC e agir de maneira precoce para evitar piora no quadro clínico do paciente.

Outras complicações típicas da CRVM, que podem surgir no período pós-operatório, foram descritas na literatura e também encontradas nos dados levantados nesta pesquisa, como a presença de instabilidade hemodinâmica, dor e angina ${ }^{(9)}$.

Segundo a literatura, os principais cuidados de enfermagem para o paciente cardíaco em pós-operatório imediato devem ser a manutenção do débito cardíaco, a diminuição/ausência de dor, perfusão tissular adequada, integridade tecidual, equilíbrio hidroeletrolítico e controle da glicemia, manutenção da ventilação e oxigenação adequadas, precauções e medidas para redução de risco de infecção, comunicação, redução da ansiedade do paciente e de seus familiares, atendendo às necessidades do autocuidado, e fornecimento de aporte nutricional $\operatorname{adequado}^{(3,8-10)}$.

No pós-operatório de cirurgia cardíaca, a complicação neurológica é multifatorial, incluindo fatores de risco pré, intra e pós-operatórios, como idade, sexo e doenças neurológicas prévias ${ }^{(7)}$. Dentre as possíveis complicações neurológicas pós CRVM, destaca-se o Acidente Vascular Cerebral (AVC) com afasia, alteração de comportamento, alteração de conduta, crise de abstinência, agitação, apatia e confusão mental ${ }^{(7)}$. De acordo com a literatura, os pacientes com complicações neurológicas necessitam receber os seguintes cuidados no período pós-operatório, quando indicados: verificação da responsividade, orientação ao paciente no tempo e no espaço e utilização da Escala de Coma de Glasgow ${ }^{(8)}$.

Dentre as principais complicações pulmonares, destacam-se a atelectasia, a pneumonia, o derrame pleural, os distúrbios ventilatórios restritivos, a paralisia ou paresia do nervo frênico e a hipoxemia ${ }^{(17)}$. A exposição do paciente, ventilação mecânica prolongada, sobrecarga volêmica iatrogênica, fazem dos pulmões foco frequente de complicações $^{(18)}$. As complicações pulmonares registradas nos prontuários, como tempo de ventilação mecânica superior a 24 horas e insuficiência respiratória, foram as que mais se assemelharam aos estudos publicados até o momento.

Frente a essas complicações, é necessária maior vigilância ao estado hemodinâmico e condição ventilatória. A mudança de decúbito, por exemplo, é uma forma de precaução ao acúmulo de secreções e colapso dos alvéolos. Assim, torna-se necessária a melhoria da amplitude respiratória, monitoração da oximetria de pulso, manutenção da frequência respiratória normal, avaliação da causa da alteração e criação de medidas para melhorar o padrão respiratório ${ }^{(8)}$. No que diz respeito às complicações renais, em paralelo a outro estudo, destaca-se a Insuficiência Renal Aguda (IRA) ${ }^{(19)}$. Na IRA ocorre uma deterioração repentina da função renal, com perda da capacidade dos rins em realizar a excreção 
das escórias nitrogenadas e manter o equilíbrio hidroeletrolítico ${ }^{(20)}$.

Diante das possíveis complicações renais existentes, é importante que o enfermeiro faça o controle do balanço hídrico e o monitoramento minucioso da função renal do paciente, observando sempre as pequenas elevações na taxa de depuração de creatinina, comprovada como indicador mais autêntico da redução da filtração glomerular, a fim de moderar ou retroceder tais agravamentos. Também são atribuições do enfermeiro realizar a avaliação do estado hemodinâmico do paciente, tais como sinais vitais, perfusão periférica, débito urinário e administrar, quando prescrito pelo médico, infusões intravenosas para reversão da hipotensão sistêmica ${ }^{(21)}$.Pacientes submetidos às cirurgias cardíacas comumente apresentam, no pós-operatório, infecções no trato respiratório e também outros tipos de infecções em consequência do próprio procedimento cirúrgico, extremamente invasivo, e das condutas no pós-operatório ${ }^{(22)}$. A infecção de sítio cirúrgico (ISC) ocupa a terceira posição entre todas as infecções relacionadas à assistência a saúde e compreende $14 \%$ a 16\% das infecções encontradas nos pacientes hospitalizados, resultado que difere dos encontrados nas cirurgias avaliadas ${ }^{(23)}$.

As complicações infecciosas após cirurgias cardíacas limpas acontecem em até 3,5\% dos pacientes, sendo as principais a mediastinite, infecção no sítio de retirada da veia safena, endocardite, infecção esternal, infecção de ferida operatória torácica, sepse, infecções pulmonares, infecções em sítios de acessos vasculares, infecções de trato urinário, infecções de trato gastrintestinal $^{(5)}$.

Os profissionais de saúde, em especial os da enfermagem, devem atentar para prevenção de infecções cruzadas durante os procedimentos invasivos. Medidas simples ajudam na prevenção da transmissão de patógenos, tais como lavagem das mãos, uso adequado dos equipamentos de proteção individual, manutenção das técnicas assépticas, manutenção correta de roupas e equipamentos contaminados, limpeza do ambiente e alojamento privativo de pacientes que possam contaminar o ambiente, técnica asséptica na realização de procedimentos e observação dos sinais de infecção em dispositivos invasivos ${ }^{(3,6)}$.

A equipe de enfermagem é a base fundamental do cuidado, pois é ela quem lida diariamente com o paciente. A atuação dos enfermeiros diante das complicações pós-operatórias cardíacas pode ser eficaz, desde que esses profissionais possuam recursos materiais e humanos adequados, além de conhecimento e habilidade, adquiridos por meio de constantes treinamentos e capacitações ${ }^{(9)}$.

Para todas as complicações pós-operatórias listadas, os cuidados de enfermagem indicados pela literatura são mais abrangentes do que aqueles identificados nos prontuários dos pacientes pesquisados, o que não significa que não tenham sido realizados, mas indica um possível sub-registro do serviço de enfermagem nos prontuários. A ausência da sistematização da assistência de enfermagem perioperatória (SAEP) nas unidades de cuidado também contribui para a invisibilidade de toda a assistência ofertada ao paciente. Destaca-se que a SAEP organiza o processo de trabalho do enfermeiro e permite a avaliação individualizada do paciente com a identificação de diagnósticos de enfermagem reais e de risco que possibilitam a prescrição de intervenções de enfermagem de acordo com as necessidades avaliadas e, assim, a prevenção de danos decorrentes das complicações.

Apesar das limitações do estudo, os resultados encontrados ajudaram a apontar as principais complicações no pós-operatório da CRVM e os cuidados de enfermagem mais ofertados (registrados) aos pacientes, bem como os fatores de riscos que podem ter contribuído para o surgimento de possíveis complicações.

\section{Conclusão}

O estudo permite concluir que a monitoração dos sinais vitais, o reconhecimento da alteração da pressão sanguínea, a monitoração do equilíbrio de líquidos e dos padrões respiratórios para bradipneia, taquipneia e hiperventilação foram 
os cuidados de enfermagem mais ofertados aos pacientes no período pós-operatório da CRVM.

Ressalta-se que estudos referentes ao tema são importantes de serem abordados e discutidos, o que permite contribuir para a qualidade do atendimento ao paciente. Além disso, pode colaborar também na prevenção de futuros eventos relacionados à história clínica do paciente ou não.

Diante disso, o enfermeiro capacitado e ciente da realidade em que vivencia tem mais oportunidades de contribuir para a melhoria do prognóstico do paciente no pós-operatório. Ao conhecer essas complicações nos períodos operatórios, é possível desenvolver um raciocínio clínico em torno da situação estabelecida. Dessa forma, há possibilidade de implementar planos de cuidados individuais que contemplem o paciente de forma integral.

\section{Colaborações:}

1. concepção, projeto, análise e interpretação dos dados: Liliane de Lourdes Teixeira Silva, Adriana Ferreira Silva, Jessica Campos Daniel, Ana Flávia Leite Andrade e Emylle Thayssa Mendonça Santos;

2. redação do artigo, revisão crítica relevante do conteúdo intelectual: Liliane de Lourdes Teixeira Silva, Luciana Regina Ferreira da Mata, Adriana Ferreira Silva, Jessica Campos Daniel, Ana Flávia Leite Andrade e Emylle Thayssa Mendonça Santos

3. aprovação final da versão a ser publicada: Liliane de Lourdes Teixeira Silva.

\section{Referências}

1. Pereira JMV, Cavalcanti ACD, Santana RF, Cassiano KM, Queluci GC, Guimarães TCF. Diagnósticos de enfermagem de pacientes hospitalizados com doenças cardiovasculares. Esc. Anna Nery [Internet]. 2011 [cited 23 Oct 2015];15(4):737-45 Available from: http://www.scielo.br/pdf/ean/ v15n4/a12v15n4.pdf

2. Brasil. Ministério da Saúde. Secretaria de Atenção à Saúde. Departamento de Atenção Especializada e Temática. Diretrizes clínicas para o cuidado ao paciente com doença renal crônica - DRC no sistema único de saúde. Brasília; 2014.

3. Lira ALBC, Araújo WM, Souza NTC, Frazão CMFQ, Medeiros ABA. Mapeamento dos cuidados de enfermagem para pacientes no pós-operatório de cirurgia cardíaca. Rev Rene [Internet]. 2012 [cited 2015 Oct 1];13(5):1171-81. Available from: http:// www.revistarene.ufc.br/revista/index.php/revista/ article/viewFile/241/pdf

4. Santos NP, Rocha AKF, Pereira MP, Borges DL, Nina VJS, Costa MAG, et al. Efeitos de diferentes níveis de peep no pós-operatório imediato de revascularização miocárdica em pacientes obesos. ASSOBRAFIR C [Internet]. 2012 [cited 2015 Oct 9];4(3):19-28. Available from: http://www.uel.br/ revistas/uel/index.php/rebrafis/article/view/15489

5. Soares GMT, Ferreira DCS, Gonçalves MPC, Alves TGS, David FL, Henriques KMC, et al. Prevalência das principais complicações pós-operatórias em cirurgias cardíacas. Rev bras cardiol [Internet]. 2011 [cited 2015 Oct 3];4(3):139-46. Available from: http://sociedades. cardiol.br/socerj/revista/2011_03/a_2011_v24_ n03_01prevalencia.pdf

6. Antônio IHF, Barroso TL, Cavalcante AMRZ, Lima LR. Qualidade de vida dos cardiopatas elegíveis à implantação de marca-passo cardíaco. Rev enferm UFPE on line [Internet]. 2010 [cited 2021 Nov 16];4(2):647-57. Available from: http:// www.researchgate.net/publication/47498477_ Quality_of_life_of_cardiac_patients_eligible_for_ implantation_of_cardiac_pacemakers

7. Carvalho ARS, Matsuda L.M, Carvalho MSS, Almeida RMSSA, Schneider DSLG. Complicações no pós-operatório de revascularização miocárdica. Ciênc cuid saúde [Internet]. 2006 [cited 2015 Oct 3];5(1):50-9. Available from: http://eduem. uem.br/ojs/index.php/CiencCuidSaude/article/ viewArticle $/ 5111$

8. Maia MA, Sade PMC. Cuidados de enfermagem no pós-operatório imediato de revascularização do miocárdio. Rev Eletrônica Faculdade Evangélica Paraná [Internet]. 2012 [cited 2015 Oct 20];2(3):1831. Available from: http://www.fepar.edu.br/ revistaeletronica/index.php/revfepar/article/ view/53

9. Teles AMC, Nogueira EC, Melo DKDS. A atuação do enfermeiro nas complicações pós-operatórias cardíacas imediatas em instituições hospitalares de Aracaju-SE. ICSA [Internet]. 2015 [cited 2015 Oct 5];3(2):19-28. Available from: https://periodicos. set.edu.br/index.php/saude/article/view/1752 
10. Duarte SCM, Stipp MAC, Mesquita MGR. O cuidado de enfermagem no pós-operatório de cirurgia cardíaca: um estudo de caso. Esc Anna Nery. 2012 Oct/Dec;16(4):657-65.

11. Araujo NR, Araujo RA, Oliveira RC, Bezerra SMMS. Complicações pós-operatórias em pacientes submetidos a cirurgia de revascularização miocárdica. J Nurs UFPE on line [Internet]. 2013 [cited 2017 May 30];7(5):1301-10. Available from: http://www.revista.ufpe.br/revistaenfermage m/ index.php/revista/article/view/4103/pdf_2484

12. Lima FET, Araújo TL, Lopes MVO, Silva LF, Monteiro ARM, Oliveira SKP. Fatores de risco da doença coronariana em pacientes que realizaram revascularização miocárdica. RENE rev min enferm [Internet]. 2012 [cited 2015 Oct 20];13(4):853-60. Available from: http://www.revistarene.ufc.br/ revista/index.php/revista/article/view/1080

13. Laizo A, Delgado FEF, Rocha GM. Complicações que aumentam o tempo de permanência na unidade de terapia intensiva na cirurgia cardíaca. Rev bras cir cardiovasc [Internet]. 2010 [cited 2015 Oct 18];25(2):166-71. Available from: http://www.scielo.br/scielo.php?script=sci_ arttext\&pid=S0102-76382010000200007

14. Kubrusly LF. Fatores de risco em cirurgias cardíacas nos septuagenários. São Paulo: Portal do coração; 2010 [cited 2015 Oct 9]. Available from: http://portaldocoracao.uol.com.br/cirurgiacardiaca/fatores-de-risco-em-cirurgias-cardiacasnos-septuagenarios

15. Araújo NR, Araújo RA, Bezerra SMMS. Repercussão do sobrepeso e da obesidade no pós-operatório da cirurgia de revascularização miocárdica. Rev Esc Enferm USP [Internet]. 2014 [cited 2015 Oct 13];48(2):p.236-41. Available from: http:// www.scielo.br/pdf/reeusp/v48n2/pt_0080-6234reeusp-48-02-236.pdf

16. Torrati FG, Dantas RAS. Circulação extracorpórea e complicações no período pós-operatório imediato de cirurgias cardíacas. Acta Paul Enferm. 2012;25(3):340-5.

17. Schmitz FS, Pascotini FS, Trevisan SRC, Albuquerque IM, Trevisan ME. Comparação de dois protocolos de espirometria de incentivo no pós-operatório de cirurgia de revascularização do miocárdio: estudo piloto. Saúde (Santa Maria). 2015 jul/ dez;41(2):129-38.
18. Cavalli F, Nohama P. Novo dispositivo EPAP subaquático no pós-operatório de cirurgia de revascularização do miocárdio. Fisioter Mov [Internet]. 2013 [cited 2015 Oct 9];26(1):37-45. Available from: http://www.scielo.br/scielo.php?pid=S0103$51502013000100004 \&$ script=sci_arttext

19. Carvalho MRM, Silva NAS, Oliveira GMM, Klein CH. Complicações e tempo de internação na revascularização miocárdica em hospitais públicos no Rio de Janeiro. Rev bras ter Intensiva [Internet]. 2011 [cited 2015 Sept 27];23(3):312-20. Available from: http://www.scielo.br/scielo.php?pid=S0103507X2011000300009\&script=sci_arttext

20. Kulaylat MN, Dayton MT. Complicações cirúrgicas. In: Townsend CM, Beauchamp RD, Evers BM, Mattox KL. Sabinston. Tratado de cirurgia: a base biológica da prática cirúrgica moderna. 18a ed. Rio de Janeiro: Elsevier; 2010. p. 327-33.

21. Graça Júnior CAGG, Mendes JR, Dourado GOL, Rodrigues EM, Araújo RA, Queiroz AAFLN. Infecções em pacientes no pós-operatório em cirurgia cardíaca: uma revisão integrativa. Rev Prev Infecç Saúde [Internet]. 2015 [cited 2015 Sept 30];1(1):59-73. Available from: http://www.ojs. ufpi.br/index.php/nupcis/article/view/3173

22. Magedanz EH, Bodanese LC, Guaragna JCVC, Albuquerque LC, Martins V, Minossi SD, et al. Elaboração de escore de risco para mediastinite pós-cirurgia de revascularização do miocárdio. Rev bras cir cardiovasc [Internet]. 2010 [cited 2015 Oct 20];25(2):154-9. Available from: http://www.scielo.br/ scielo.php?pid $=$ S010276382010000200005\&script $=$ sci_ abstract\&tlng=pt

23. Tiveron MG, Fiorelli AI, Mota EM, Mejia OAV, Brandão CMA, Dallan LAO, et al. Fatores de risco pré-operatórios para mediastinite após cirurgia cardíaca: análise de 2768 pacientes. Rev bras cir cardiovasc [Internet]. 2012 [cited 2015 Oct 3];27(2):203-10. Available from: http://www.scielo.br/scielo.php?script=sci_ arttext\&pid=S0102-76382012000200007

Recebido: 16 de novembro de 2016

Aprovado: 21 de agosto de 2017

Publicado: 19 de outubro de2017 